\title{
Surgery for Severe Ischemic Mitral Regurgitation
}

TO THE EDITOR: In 2009, Magne et al. ${ }^{1}$ summarized numerous studies showing that simple ring repair of severe ischemic regurgitation associated with moderate or severe tethering would prematurely fail. They concluded that comprehensive imaging was necessary to tailor the intervention to the individual patient. Subsequently, a multi-institutional laboratory trial showed that more complex annular and subvalvular repair was required to optimize remodeling and prevent recurrent regurgitation. ${ }^{2}$ Complex repair is also supported clinically. ${ }^{3,4}$

Nonetheless, Acker et al. (Jan. 2 issue) ${ }^{5}$ used a simple ring for all repairs, even though tethering was required for enrollment. Mitral intervention was guided solely by regurgitant grade. Conducting a blind, randomized trial in which all patients with ischemic regurgitation were assigned to simple ring repair or replacement is akin to randomly assigning all patients with lung cancer to lobectomy or pneumonectomy, regardless of the stage of disease. The trial does not support the authors' conclusion that replacement is superior to repair. They simply reaffirm that simple ring repairs fail when moderate or severe tethering is present.

Axial echocardiography greatly facilitates the stratification of disease severity and the identification of the individualized techniques required to optimize remodeling, provide freedom from recurrence, and increase the likelihood of survival (Fig. 1). ${ }^{4}$ Detailed image guidance is essential to achieve the best possible results.

Daniel H. Drake, M.D.

Karen G. Zimmerman, B.S., R.D.C.S. Cynthia D. Nichols, Ph.D.

Munson Medical Center

Traverse City, MI

daniel.h.drake@gmail.com

No potential conflict of interest relevant to this letter was reported.

1. Magne J, Sénéchal M, Dumesnil JG, Pibarot P. Ischemic mitral regurgitation: a complex multifaceted disease. Cardiology 2009;112:244-59.

2. Szymanski C, Bel A, Cohen I, et al. Comprehensive annular and subvalvular repair of chronic ischemic mitral regurgitation improves long-term results with the least ventricular remodeling. Circulation 2012;126:2720-7.

3. Borger MA, Murphy PM, Alam A, et al. Initial results of the chordal-cutting operation for ischemic mitral regurgitation. J Thorac Cardiovasc Surg 2007;133:1483-92.

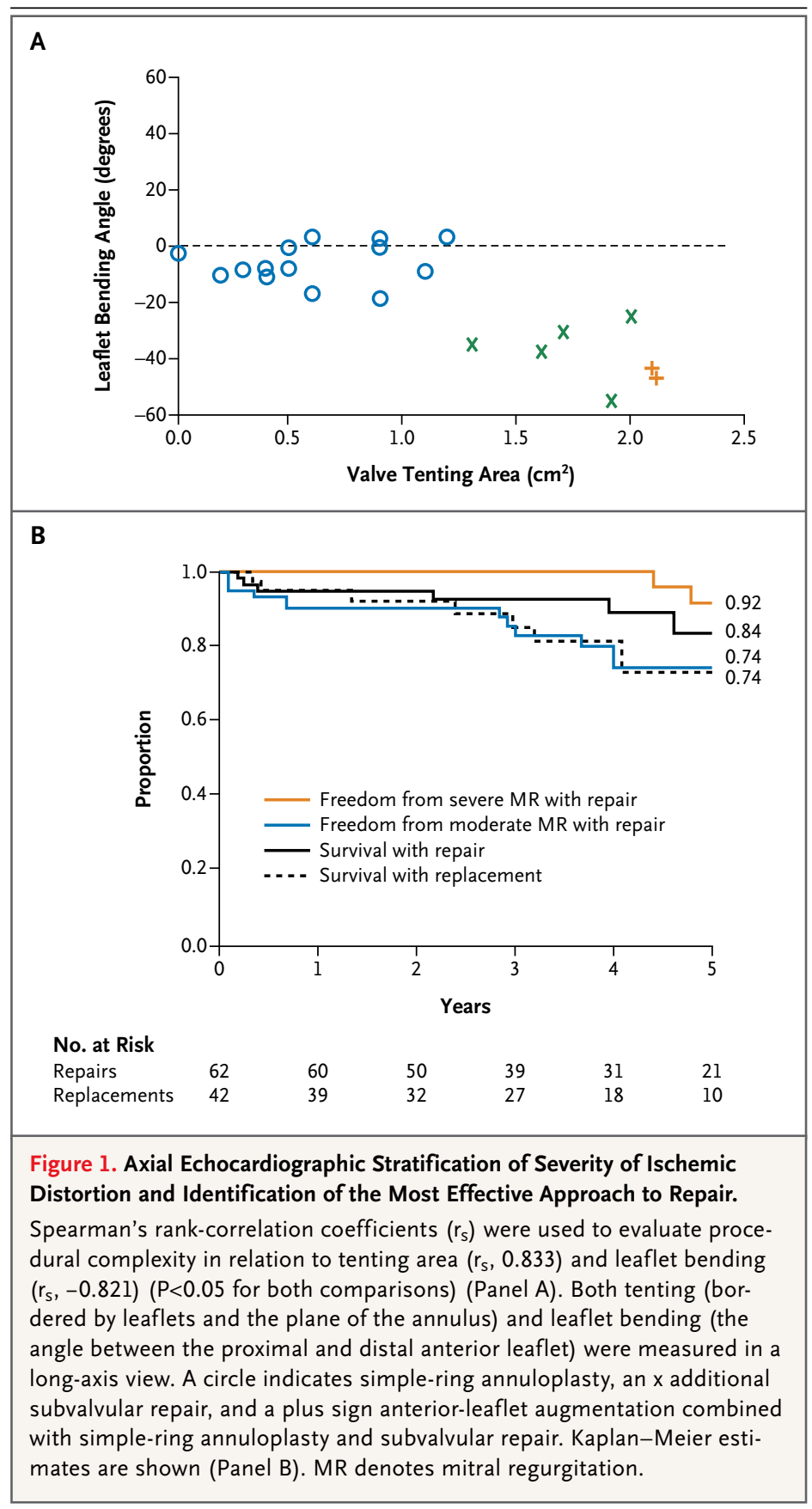

4. Drake DH, Zimmerman KG, Hepner AM, Nichols CD. Echoguided mitral repair. Circ Cardiovasc Imaging 2014;7:132-41. 5. Acker MA, Parides MK, Perrault LP, et al. Mitral-valve repair versus replacement for severe ischemic mitral regurgitation. N Engl J Med 2014;370:23-32.

DOI: $10.1056 /$ NEJMc1401885 
TO THE EDITOR: Acker et al. report no significant difference between mitral-valve annuloplasty and replacement regarding left ventricular reverse remodeling and 12-month survival in patients with severe ischemic mitral regurgitation, which was defined as an effective regurgitant orifice of more than $40 \mathrm{~mm}^{2}$. However, because of the often hemielliptical shape of the mitral-regurgitation convergence zone, its use may lead to underestimation of the severity of mitral regurgitation. Severe ischemic mitral regurgitation should be considered when the effective regurgitant orifice is greater than $20 \mathrm{~mm}^{2}$. Consequently, a large number of patients probably had massive mitral regurgitation, limiting the potential effects of mitral-valve annuloplasty. When the effective regurgitant orifice was not quantifiable, severe mitral regurgitation was defined according to an integrative approach that included the ratio of jet area to the left atrial area, which is no longer recommended, ${ }^{1}$ or an approach that relied on vena contracta jet width without a specified cutoff value.

The authors do not mention the extent of the undersizing (i.e., one or two sizes) when they describe the mitral-valve annuloplasty procedure. Furthermore, to be effective, annuloplasty should target a leaflet-coaptation width of $8 \mathrm{~mm}$ or more. ${ }^{2}$ Although mitral-valve annuloplasty is not necessarily the best possible surgical approach for patients with ischemic mitral regurgitation, the results of the present study need to be interpreted carefully.

Julien Magne, Ph.D.

Centre Hospitalier Universitaire Limoges Limoges, France

Luc A. Pierard, M.D., Ph.D.

University of Liege

Liege, Belgium

jul.magne@yahoo.fr

No potential conflict of interest relevant to this letter was reported.

1. Lancellotti P, Tribouilloy C, Hagendorff A, et al. Recommendations for the echocardiographic assessment of native valvular regurgitation: an executive summary from the European Association of Cardiovascular Imaging. Eur Heart J Cardiovasc Imaging 2013;14:611-44.

2. Braun J, van de Veire NR, Klautz RJ, et al. Restrictive mitral annuloplasty cures ischemic mitral regurgitation and heart failure. Ann Thorac Surg 2008;85:430-7.

DOI: 10.1056/NEJMc1401885

TO THE EDITOR: Acker et al., reporting for the Cardiothoracic Surgical Trials Network, describe the results of a multicenter, randomized trial to evaluate the relative benefits and risks of valve repair versus replacement in patients with severe ischemic mitral regurgitation. No significant difference between the treatment groups was observed in left ventricular volume or survival at 12 months. However, whereas significant recurrent ischemic mitral regurgitation developed in more than $30 \%$ of the patients in the repair group, the patients in this group who did not have recurrent ischemic mitral regurgitation realized a $22.6 \%$ reduction in left ventricular volume versus a $6.8 \%$ reduction in the valve-replacement group. These data suggest a large potential benefit of valve repair if the effects of recurrent ischemic mitral regurgitation can be limited. The maturation of percutaneous valve-replacement technology that has occurred since the study's inception offers the potential for a new therapeutic model for these high-risk patients: all such patients should receive surgical annuloplasty, with recurrent ischemic mitral regurgitation treated with percutaneous valve-in-ring procedures. ${ }^{1-3}$ This clinical strategy would maximize the beneficial effects of repair, eliminate the early morbidity associated with replacement, and neutralize the effects of recurrent ischemic mitral regurgitation.

Robert C. Gorman, M.D.

University of Pennsylvania

Philadelphia, PA

gormanr@uphs.upenn.edu

Matthew J. Gillespie, M.D.

Children's Hospital of Philadelphia

Philadelphia, PA

Joseph H. Gorman III, M.D.

University of Pennsylvania

Philadelphia, PA

Dr. J.H. Gorman reports serving as the principal investigator for the Strategies for Transcatheter Mitral Valve Replacement trial, supported by a grant from the National Institutes of Health (RO1HL115323), and Drs. R.C. Gorman and Gillespie report serving as coinvestigators for this trial; Drs. J.H. Gorman, R.C. Gorman, and Gillespie also report that they are inventors of devices related to percutaneous valve-replacement technology for which patents are pending and that the patents are assigned to the University of Pennsylvania. No other potential conflict of interest relevant to this letter was reported.

1. Shuto T, Kondo N, Dori Y, et al. Percutaneous transvenous Melody valve-in-ring procedure for mitral valve replacement. J Am Coll Cardiol 2011;58:2475-80.

2. Kondo N, Shuto T, McGarvey JR, et al. Melody valve-in-ring procedure for mitral valve replacement: feasibility in four annuloplasty types. Ann Thorac Surg 2012;93:783-8.

3. Himbert D, Descoutures F, Brochet E, et al. Transvenous mitral valve replacement after failure of surgical ring annuloplasty. J Am Coll Cardiol 2012;60:1205-6.

DOI: $10.1056 /$ NEJMc1401885 
THE AUTHORS REPLY: The primary aim of our trial was to compare the most common mitralvalve repair procedure performed worldwide for severe ischemic mitral regurgitation (which involves placement of an undersized annuloplasty ring) with chord-sparing valve replacement. Contrary to the comments of Drake et al., a subvalvular procedure for severe tethering was a protocoldefined surgical option. Moreover, our conclusion was not that replacement is better than repair but rather that both methods are equivalent in degree of reverse remodeling, adverse events, quality of life, and survival at 1 year. The repair group had a $32 \%$ incidence of recurrent moderate-tosevere mitral regurgitation, which is consistent with the published literature. The planned 2-year follow-up should offer further insights into the effects of recurrent mitral regurgitation. As Drake et al. point out, although there is limited and preliminary experience with the use of tethering to guide mitral-valve repair and the severing of chords as an adjunct to mitral-valve ring annuloplasty, these approaches are less well validated than simple ring repair. Planned subanalyses will help determine the echocardiographic and clinical measures that predict recurrence.

Magne and Pierard advocate defining severe ischemic mitral regurgitation as an effective regurgitant orifice area of $0.2 \mathrm{~cm}^{2}$ rather than $0.4 \mathrm{~cm}^{2}$. We used the integrated approach to grade severe mitral regurgitation that is recommended in valve guidelines by both the American College of Cardiology-American Heart Association and the European Society of Cardiology. ${ }^{1,2}$ This approach incorporates multiple variables without overreliance on a single measure. The mean effective regurgitant orifice areas for the treatment groups were $0.39 \mathrm{~cm}^{2}$ and $0.40 \mathrm{~cm}^{2}$, which appropriately reflect a lower range for effective regurgitant orifice area for ischemic mitral regurgitation. Moreover, the average ring size used in our trial was $28.4 \pm 1.9 \mathrm{~mm}$ for men and $27.2 \pm 1.6 \mathrm{~mm}$ for women, indicating that there was substantial undersizing, with more than $90 \%$ of patients in the repair group receiving a complete ring.
Gorman et al. point out that there was substantial reverse remodeling among the patients in the repair group who did not have recurrent moderate or severe mitral regurgitation (a $22.6 \%$ reduction in left ventricular volume, vs. $6.8 \%$ in the replacement group). They propose that all patients should undergo repair and that those in whom postoperative moderate or severe mitral regurgitation develops ( $32 \%$ in our study) should undergo secondary percutaneous mitral-valve replacement. A less speculative and less aggressive approach would be to use predictive models of recurrent mitral regurgitation and, in patients with a high likelihood of recurrence, to use replacement or a more complex repair technique that specifically addresses leaflet tethering. Our trial clearly shows that mitral-valve replacement with complete chordal sparing is an acceptable and safe treatment option in patients with severe ischemic mitral regurgitation.

Michael A. Acker, M.D.

University of Pennsylvania School of Medicine Philadelphia, PA

Annetine C. Gelijns, Ph.D.

Icahn School of Medicine at Mount Sinai

New York, NY

annetine.gelijns@mssm.edu

Irving L. Kron, M.D.

University of Virginia Medical Center

Charlottesville, VA

Since publication of their article, the authors report no further potential conflict of interest.

1. Bonow RO, Carabello BA, Chatterjee K, et al. 2008 Focused update incorporated into the ACC/AHA 2006 guidelines for the management of patients with valvular heart disease: a report of the American College of Cardiology/American Heart Association Task Force on Practice Guidelines (Writing Committee to Revise the 1998 Guidelines for the Management of Patients With Valvular Heart Disease): endorsed by the Society of Cardiovascular Anesthesiologists, Society for Cardiovascular Angiography and Interventions, and Society of Thoracic Surgeons. Circulation 2008;118(15):e523-e661.

2. The Joint Task Force on the Management of Valvular Heart Disease of the European Society of Cardiology (ESC), European Association for Cardio-Thoracic Surgery (EACTS). Guidelines on the management of valvular heart disease (version 2012). Eur Heart J 2012;33:2451-96.

DOI: 10.1056/NEJMc1401885

\section{Increase in Left Ventricular Assist Device Thrombosis}

TO THE EDITOR: The number of implantations of increasing worldwide, and the article by Starling left ventricular assist devices (LVADs) is greatly et al. (Jan. 2 issue) ${ }^{1}$ is of major interest in the 